\title{
THE HEEGAARD GENUS OF MANIFOLDS OBTAINED BY SURGERY ON LINKS AND KNOTS
}

\author{
BRADD CLARK \\ Department of Mathematics \\ University of Southwestern Louisiana \\ Lafayette, Louisiana 70504 \\ U.S.A.
}

(Received January 15, 1979)

ABSTRACT. Let $\mathrm{L} \subset \mathrm{S}^{3}$ be a fixed link. It is shown that there exists an upper bound on the Heegaard genus of any manifold obtained by surgery on L. The tunnel number of $L, T(L)$, is defined and used as an upper bound. If $K^{\prime}$ is a double of the knot $K$, it is shown that $T\left(K^{\prime}\right) \leq T(K)+1$. If $M$ is a manifold obtained by sungery on a cable link about $K$ which has $n$ components, it is shown that the Heegaard genus of $M$ is at most $T(K)+n+1$. 1980 MATHENATICS SUBJECT CLASSIFICATION CODES. PrimarY 57M25, 57N10. KEY WORDS AND PHRASES. Links, Knots, Heegaard genus, 3-manifold.

\section{INIRODUCTION.}

Lickorish in [5] demonstrated that all compact connected orientable 3-manifolds without boundary could be represented as a result of surgery on a link in $s^{3}$. Since then, considerable work has been done on the so-called closed 3-manifolds by studying various knots and links in $s^{3}$. 
Both in [3] and [8] a relationship between a knot and the Heegaard splitting of manifolds abtained by surgery on that knot was hinted at. In [2], Dibner showed that the Mazur homology sphere (see [6]) has a genus-2 Heegaard splitting. A similar process was used in [1] to show that $\mathrm{n}+1$ is an upper bound to the genus of a manifold obtained by surgery on a torus link with $n$ components. The purpose of this paper is to extend these techniques to other knots and links and thereby obtain a bound on the Heegaard genus of manifolds obtained by surgery on those links and knots.

If $x$ is a point set, we shall use int(X) for the interior of $x, c l(X)$ for the closure of $x$, and $\partial x$ for the boundary of $x$. The genus of a manifold is defined to be the minimal genus of a Heegaard splitting of the manifold. Let $X$ be a polyhedron contained in the P.I. $n$-manifold $M . N(X) \subset M$ is called a regular neighborhood of $X$ in $M$ if $X \subset N(X)$ and $N(X)$ is an $n$-manifold which can be simplicially collapsed to $\mathrm{x}$.

This paper deals with piecewise linear topology. As such, all manifolds are considered to be simplicial and maps to be piecewise linear. This will be assumed as additional hypotheses throughout the paper.

\section{THE TUNNET NUMBER.}

Let $L$ be a link in $\mathbb{R}^{3}$. Let $P$ be any plane and $p: \mathbb{R}^{3} \rightarrow P$ the orthogonal projection. $P$ is regular for $L$ if for every $x \in P, P^{-1}(x) \cap L$ consists of at most two points; and if $\mathrm{p}^{-1}(\mathrm{x}) \cap \mathrm{L}$ does contain two points, neither is a vertex of $L$. The crossing number for $L, C(L)$, is the minimum number of double-points in any regular projection of any link $L^{\prime}$ of the same link type as $\mathrm{L}$.

A link $L=L_{1} U L_{2}$ is splittable if there exist disjoint 3-cells $B_{1}$ and $B_{2}$ with $L_{1} \subset$ int $B_{1}$ and $L_{2} \subset$ int $B_{2}$. Thus if $L$ is any link we can write $L=L_{1} U \ldots U_{n}$ where $L_{i} \subset$ int $B_{i}, B_{i} \cap B_{j}=\varnothing$ for $i \neq j$ and $L_{i}$ is unsplittable for $1 \leq \mathrm{i} \leq \mathrm{n}$. 
Let $\mathrm{L}$ be a link in $\mathrm{S}^{3}$ and $\mathrm{N}(\mathrm{L})$ a regular neighborhood of $\mathrm{L}$ in $\mathrm{S}^{3}$. We associate with $L$ the manifold $C, c l\left(S^{3}-N(L)\right)$.

DEFINITION. $t \subset C$ is a tumnel if $t$ is a $3-c e l l$ and $t \cap \partial C$ is a pair of disjoint 2-cells.

THEOREM 2.1. If $M$ is a 3-manifold obtained by surgery on $L=L_{1} U \ldots U_{L^{\prime}}$ then the genus of $M$ is at most $\sum_{i=1}^{n} c\left(L_{i}\right)+n$.

PROOF: $M$ can be written as $M_{1} \# \ldots \# M_{n}$ where $M_{i}$ is the manifold obtained by surgery on the link $L_{i}$. By [4] we know that the genus of a connected sum is the sum of the genera of the components. Thus it will suffice to show that the genus of $M_{i}$ is at most $c\left(L_{i}\right)+1$.

If $c\left(L_{i}\right)=m$, we can find a regular projection for $L_{i}$ which has $m$ double points. We can find $\ell_{1}, \ldots, l_{m}$ where $l_{j}$ is the straight line segment between the points of $L_{i}$ which form the $j$-th double point of the projection. Let $N\left(L_{i}\right)$ be a regular neighborhood of $L_{i}$ in $S^{3}$, and $C$ its associated manifold. For each $\ell_{i}$ we can find a tunnel $t_{i}$ such that $t_{i}$ is a regular neighborhood of $l_{i} \cap c$ and such that $t_{i} \cap t_{j}=\varnothing$ for $i \neq j$.

$H=\bigcup_{j=1}^{m} t U N\left(L_{i}\right)$ is a handlebody. Clearly $\bigcup_{j=1}^{m} \ell_{j} U L_{i}$ is a core for this handlebody and this core can be deformed into the plane of projection. Therefore $\mathrm{H}^{\prime}, \mathrm{cl}\left(\mathrm{S}^{3}-\mathrm{H}\right)$ is also a handlebody. Since $\mathrm{L}_{i}$ is unsplittable, the core of $\mathrm{H}$ has one component. After deforming this core into the plane, a simple Euler characteristic argument shows that the genus of $H$ is $m+1$. We note that if $N^{\prime}\left(L_{i}\right)$ is a regular neighborhood of $L_{i}$ in $M_{i}$, then $H^{\prime \prime}=\bigcup_{j=1}^{m} t_{j} U N^{\prime}\left(L_{i}\right)$ and $H^{\prime}$ form a Heegaard splitting of $M_{i}$.

The above theorem gives a rather crude estimate of the maximum genus of a manifold obtained by surgery on a link. As we shall see, in specific situations we will be able to give a better estimate. We also see that if $L$ is an unsplittable link, we can always find a set of pairwise disjoint tunnels which can convert the associated manifold to a handlebody. 
DEFTNITION. If $L$ is an unsplittable link, the tumnel number of $L, T(L)$, is the minimum number of disjoint tunnels needed to convert the associated manifold into a handlebody.

\section{SURGERY ON KNOTS.}

In [7] H.F. Trotter described a class of knots known as pretzel knots. It is clear from this description that any pretzel knot $\mathrm{K}$ can be embedded in the boundary of a cube-with-two-handles $\mathrm{H}$ in such a way that there are cutting disks $D_{1}$ and $D_{2}$ for $H$ so that $D_{1} \cap K$ and $D_{2} \cap k$ each consist of two points. Also $\mathrm{cl}\left(\mathrm{S}^{3}-\mathrm{H}\right)$ is itself a cube-with-two-handles.

PROPOSITION 3.1. If $M$ is obtained by surgery on a pretzel knot, then $M$ has Heegaard genus at most 3 .

PRDOF. We assume that the pretzel knot $\mathrm{K} \subset \partial \mathrm{H}$ is as described above. We can find an arc $\alpha_{i} \subset D_{i}$ with $\partial \alpha_{i}=k \cap D_{i}$ for $i=1$ or 2 . Without loss of generality we may assume that a regular neighborhood of the cutting disk $D_{i}$ in $H$ is of the form $D_{i} \times I$ and that $K \cap\left(D_{i} \times I\right)=\partial \alpha_{i} \times I$.

If $N(K)$ is a regular neighborhood of $K$ in $H$, then obviously $H^{\prime}=\mathrm{N}(\mathrm{K}) U\left(D_{1} \times I\right) U\left(D_{2} \times I\right)$ is a cube-with-three-handles. Let $B=c l\left(H-\left[\left(D_{1} \times I\right) U\left(D_{2} \times I\right)\right]\right)$. We note that $(K \cap B) U\left(\alpha_{1} \times\{0,1\}\right) \cup\left(\alpha_{2} \times\{0,1\}\right)$ is a simple closed curve in $\partial B=s^{2}$. Let $D$ be the disk in $B$ bounded by this simple closed curve. Then $D$ along with the two cutting disks of $\mathrm{cl}\left(\mathrm{S}^{3}-\mathrm{H}\right)$ will form a system of cutting disks for $\mathrm{cl}\left(\mathrm{S}^{3}-\mathrm{H}^{\prime}\right)$. Using the same reasoning as in Theorem 2.1, we have found a genus 3 Heegaard splitting for any manifold obtained by surgery on a pretzel knot.

PROPOSITION 3.2. If $M$ is a manifold obtained by surgery on the composite knot $K_{1} \# K_{2}$, then the genus of $M$ is at most $T\left(K_{1}\right)+T\left(K_{2}\right)+2$.

PROOF. We can find a 2-sphere $S \subset S^{3}$ with the following properties. $S$ separates $s^{3}$ into two 3-cells $B_{1}$ and $B_{2} \cdot\left(K_{1} \# K_{2}\right) \cap s$ consists of two points. If $\alpha \subset S$ is an arc connecting these two points, 
$\left[B_{1} \cap\left(K_{1} \# K_{2}\right)\right] U_{\alpha}$ is a knot of type $K_{1}$ and $\left[B_{2} \cap\left(K_{1} \# K_{2}\right)\right] U_{\alpha}$ is a knot of type $k_{2}$. Without loss of generality we may assume that $S \times I$ is a regular neighborhood of $S$ in $S^{3}$ and that $(S \times I) \cap\left(K_{1} \# K_{2}\right)=\partial \alpha \times I$. Since $K_{1}^{\prime}=\left[\left(K_{1} \# K_{2}\right) \cap \mathrm{cl}\left(B_{1}-s \times I\right)\right] U[\alpha \times\{0\}]$ is a knot of type $K_{1}$, it has tunnel number $T\left(K_{1}\right)$. We can find a Heegaard splitting of $B_{1}$ of genus $\mathrm{T}\left(\mathrm{K}_{1}\right)+1$. Likewise since $\mathrm{K}_{2}^{\prime}=\left[\left(\mathrm{K}_{1} \# \mathrm{~K}_{2}\right) \cap \mathrm{cl}\left(\mathrm{B}_{2}-\mathrm{S} \times \mathrm{I}\right)\right] \mathrm{U}[\alpha \times\{1\}]$ is a knot of type $K_{2}$ it has tunnel number $T\left(K_{2}\right)$ and we can find a Heegaard splitting of $\mathrm{B}_{2}$ of genus $\mathrm{T}\left(\mathrm{K}_{2}\right)+1$. We can connect these splittings by adding the 3-cell which is a regular neighborhood of $\alpha \times I$ in $S \times I$. This will yield a Heegaard splitting of $M$ of genus $T\left(K_{1}\right)+T\left(K_{2}\right)+2$.

PROPOSITION 3.3. If $K^{\prime}$ is a double of $K$ and $M$ is a manifold abtained by surgery on $K^{\prime}$, then $M$ has genus at most $T(K)+2$.

PROOF. Since $K^{\prime}$ is a double of $K$ we can find a singular disk $D$ with the following properties. A copy of $K$ is contained in D. $K^{\prime}$ is spanned by $D$ and the only singularity in $D$ is a single double arc $\alpha$ with $\partial \alpha \subset K^{\prime}$.

Let $N\left(K^{\prime} U_{\alpha}\right)$ be a regular neighborhood of $K^{\prime} U_{\alpha}$ in $s^{3}$. We note that $D^{\prime}=\operatorname{cl}\left(D-N\left(K^{\prime} U_{\alpha}\right)\right.$ ) is a real disk and that $N(D)$ is a solid torus with a knot of type $K$ for a core. This means that if we add $T(K)$ tumnels to $N(D)$ we can obtain a Heegaard splitting of genus $T(K)+1$. When we remove $N\left(D^{\prime}\right)$ fram $N(D)$ we will obtain a Heegaard splitting of $M$ of genus $T(K)+2$.

In addition to these specific knots, we can find a better estimate than Theorem 2.1 gives for the Heegaard genus of manifolds obtained by surgery on specific links.

4. SURGERY ON CABIE IINNKS.

THEOREM 4.1. If $M$ is a 3-manifold obtained by surgery along a cable link about $K$ with $n$ components, then $M$ has genus at most $T(K)+n+1$.

PROOF. Let $T$ be a solid torus in $S^{3}$ which has $K$ for a core. We split วT into $2 \mathrm{n}$ annuli $\left\{\mathrm{A}_{1}, \mathrm{~B}_{1}, \mathrm{~A}_{2}, \mathrm{~B}_{2}, \ldots, \mathrm{A}_{\mathrm{n}}, \mathrm{B}_{\mathrm{n}}\right\}$ each following an $(\mathrm{x}, \mathrm{Y})$ curve 
on $\partial \mathrm{T}$. Let $\partial \mathrm{T} \times \mathrm{I}$ be a collar attached to $\mathrm{T}$ with $\partial \mathrm{T}=\partial \mathrm{T} \times\{0\}$. Let $\mathrm{C}=\mathrm{cl}\left(\mathrm{S}^{3}-(\mathrm{T} U(\partial \mathrm{T} \times \mathrm{I}))\right)$. Without loss of generality, we may assume the existence of $T(K)$ tumnels $t_{1}, \ldots, t_{T(K)}$ contained in $C$ such that cl $\left(C-U_{i=l} t_{i}\right)$ is a cube-with-handles. We may also assume that $t_{i} \cap \partial C \subset \operatorname{int}\left(B_{1} \times\{1\}\right) \subset \partial T \times I$.

We can think of $M$ as being obtained by surgery on $A_{i} \times[1 / 2,1]$ where $A_{i} \times I \subset \partial T \times I$. Let $D_{i}$ be a disk in $A_{i}$ with the property that $\operatorname{cl}\left(A_{i}-D_{i}\right)$ is a disk. Iet $\alpha_{i}$ and $\beta_{i}$ be arcs in $\partial A_{i}$ with the property that $\partial A_{i} \cap\left(\operatorname{cl}\left(A_{i}-D_{i}\right)=\alpha_{i} \cup \beta_{i}\right.$. Finally, we let $N_{i}=h\left(A_{i} \times[1 / 2,1]\right) \subset M$ be the image of $A \times[1 / 2,1]$ in $M$ after surgery is performed on the cable link. Since $D_{i} \times[0,1 / 2] \subset \partial T \times I$ is a $3-c e l l$ and $\left(D_{i} \times[0,1 / 2]\right) \cap T$ and $\left(D_{i} \times[0,1 / 2]\right) \cap N_{i}$ are disks, we have that $H_{1}=T U^{n}\left[\left(D_{i} \times[0,1 / 2]\right) U N_{i}\right.$ is a cube-with-n+l-handles. We know that $\left[\begin{array}{c}U \mathrm{~T}(\mathrm{~K}) \\ \mathrm{i}=1\end{array} t_{i}\right] \bigcap^{i=1} \partial \mathrm{C}$ is a set of $2 \mathrm{~T}(\mathrm{~K})$ pairwise disjoint disks contained in int $\left(B_{1} \times\{1\}\right)$. If $t_{i} \cap \partial c=d_{i, 1} U d_{i, 2}$, we can extend $t_{i}$ to the 3-cell $t_{i}^{\prime}=t_{i} U\left(d_{i, 1} \times I\right) U\left(d_{i, 2} \times I\right)$. Then $H_{1}^{\prime}=H_{1} U_{i=1}^{T(K)} t_{i}^{\prime}$ is a cube-with-n+T(K)+1-handles.

We note that since $C$ is homeamorphic to $C_{T(K)} U_{i=1}^{n}\left(B_{i} \times I\right)$, we must have that $\mathrm{H}_{2}=\mathrm{cl}\left[\left(\mathrm{CU}_{i=1}^{\mathrm{n}}\left(\mathrm{B}_{i} \times I\right)\right)-U_{j=1}^{\mathrm{T}(\mathrm{K})} \mathrm{t}_{j}^{\prime}\right]$ is a cube-with-T(K)+l-handles. Since $\alpha_{i} \times I$ and $\beta_{i} \times I$ are disks on $\partial H_{2}$ where $\alpha_{i} \times I$ and $\beta_{i} \times I$ are contained in $\partial \mathrm{T} \times \mathrm{I}$, we have that $\mathrm{cl}\left(\mathrm{A}_{i}-\mathrm{D}_{i}\right) \times I \subset \partial \mathrm{T} \times I$ is a handle added to $\mathrm{H}_{2}$. Thus $\mathrm{M}$ - int $\mathrm{H}_{1}^{\prime}=\mathrm{H}_{2}^{\prime}$ is also a cube-with-n+T(K)+1-handles.

\section{REFERENCES}

1. Clark, B. Surgery on Links Containing a Cable Sublink, Proc. Amer. Math. Soc. (to appear).

2. Dibner, S. Heegaard Splittings for an Infinite Family of Closed Orientable 3-manifolds, Ph.D. Thesis, State University of New York at Binghamton, 1977.

3. Haken, W. Various Aspects of the Three-dimensional Poincare Problem, Topology of Manifolds, ed. J.C. Cantrell and C.H. Edwards, (Markham, 
Chicago) (1969) 140-152.

4. Haken, W. Some Results on Surfaces in 3-manifolds, Studies in Modern Topology, ed. P.J. Hilton, M.A.A. Studies in Math. Vol. 5, 39-98.

5. Lickorish, W.B.R. A Representation of Orientable Cambinatorial 3-manifolds, Ann. of Math. 76 (1962), 531-540.

6. Mazur, B.C. A Note on Some Contractible 4-manifolds, Ann. of Math. 73 (1961) 221-228.

7. Trotter, H.F. Non-invertible Knots Exist, Topology 2 (1964) 275-280.

8. Waldhausen, F. Uber Involutionen der 3-sphere, Topology 8 (1969) 81-91. 


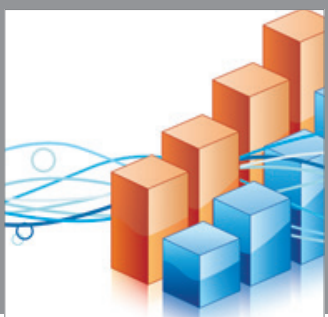

Advances in

Operations Research

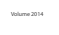

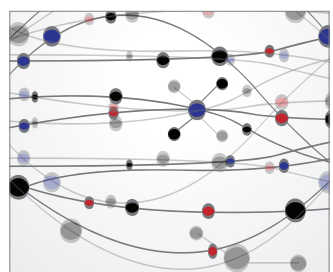

\section{The Scientific} World Journal
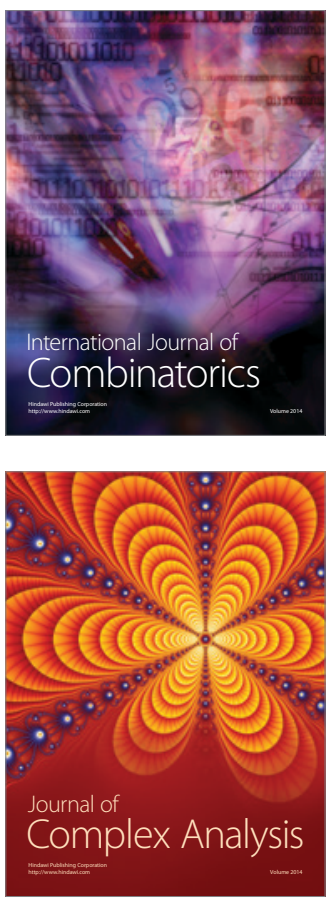

International Journal of

Mathematics and

Mathematical

Sciences
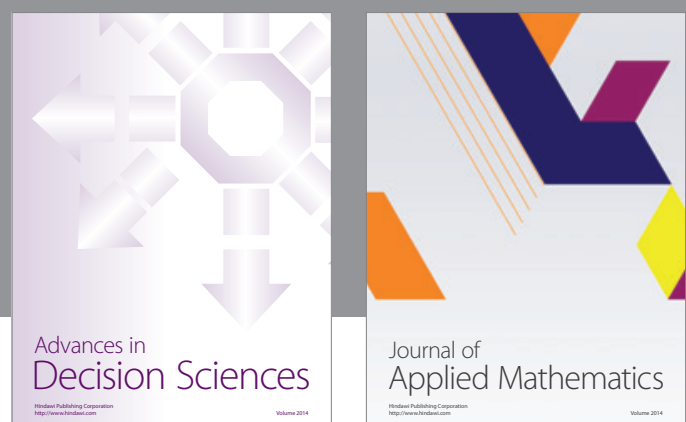

Journal of

Applied Mathematics
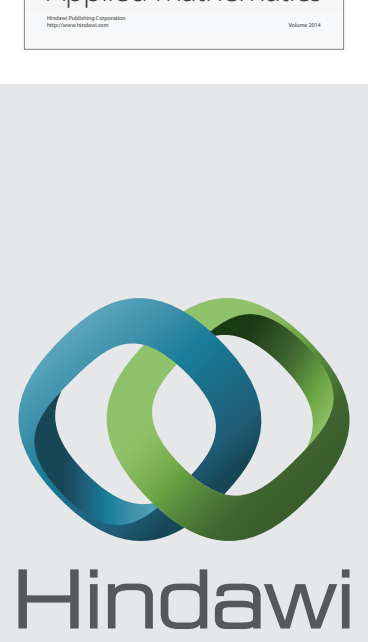

Submit your manuscripts at http://www.hindawi.com
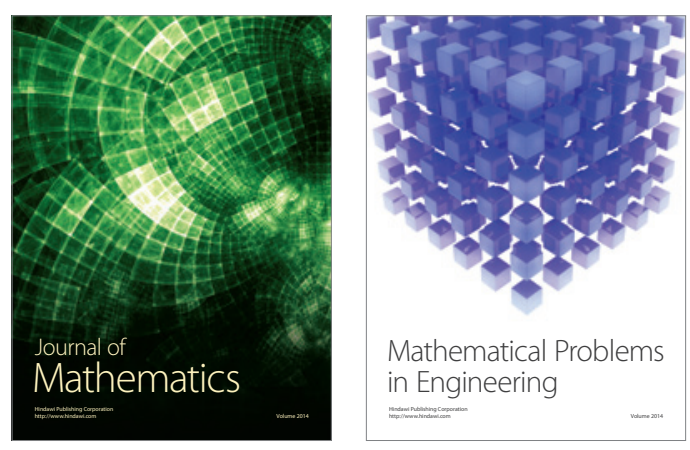

Mathematical Problems in Engineering
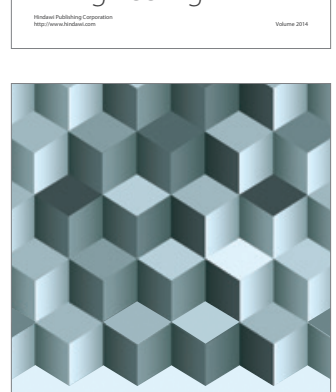

Journal of

Function Spaces
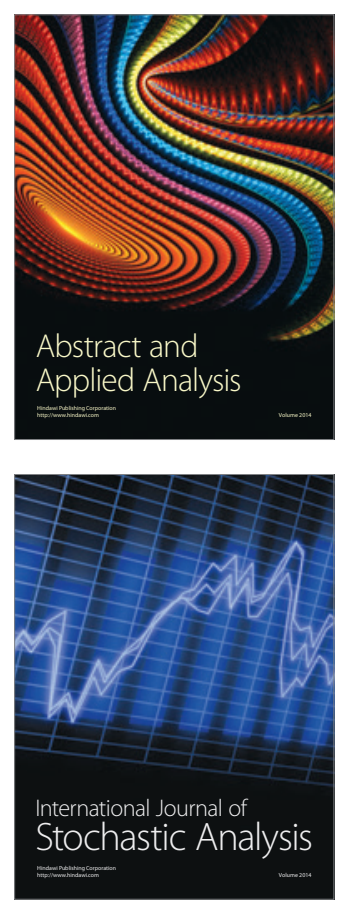

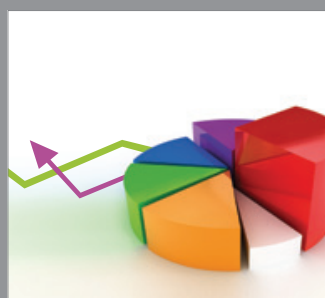

ournal of

Probability and Statistics

Promensencen
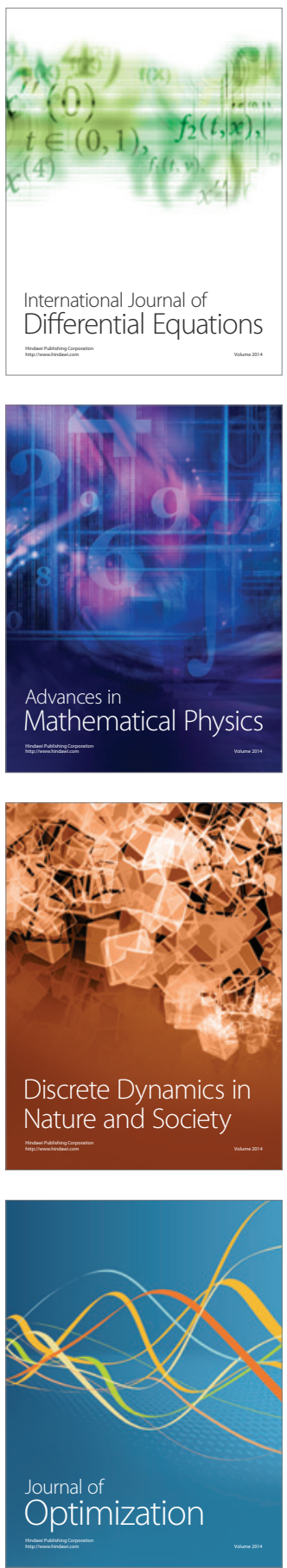